\title{
Expériences faites en Hongrie avec des installations de mesures hydrauliques ${ }^{(*)}$
}

\author{
Hungarian experiences \\ with hydraulic measuring devices \\ PAR Ö. STAROSOLSZIXY
}

INGÉNIEUR DE RECHERCHES A L'INSTITUT DE RECHERCHES DES RESSOURCES HYDRAUIQUES A BUDAPEST

\begin{abstract}
L'article décrit les conditions à remplir par les installations de mesure des débits en canaux ou en conduites, et par les ouvrages de mesures en laborutoire et in situ, notamment tubes Venturi, coudes, mesureurs proportionnels, pertuis Pikalov, canaux Parshall, canaux Venturi trapézoüdaux et ouvrages de prise secondaires. Puis il relate les expériences faites en Hongrie sur ce sujet et les perspectives futures des recherches en cours.
\end{abstract}

\begin{abstract}
The anthor discusses the requirements for flowmeasurements devices in canals or ducts, and laboratory and field measuring equipment and structures, such as Venturi tubes, elbows, flow proportioners, Pikalov sluices, Parshall flumes, trapezoidal Venturi flumes, and secondary offtakes. He also gives an account of experience acquired on the subject in Hungary and the anticipated results of present research.
\end{abstract}

\section{I. - NECESSITE ET OBJET DES INSTALLATIONS DE MESURES HYDRAULIQUES}

Il est impossible d'obtenir des mesures de débits continues suffisamment précises sans avoir recours à des installations de mesures hydrauliques. En Hongrie, ces installations sont placées sur des petits cours d'eau naturels, ainsi que sur des canaux de drainage et d'irrigation. Sur ces derniers, on peut résoudre le problème en étalonnant des ouvrages construits à l'origine pour d'autres emplois [1], mais étant donné que ces ouvrages ont été étudiés sans tenir compte de la nécessité d'effectuer des mesures, la bonne solution est encore d'utiliser des installations de mesures proprement dites.

Installations de mesures en canaux ou en conduites. - Ces installations sont tenues pour nécessaires dans n'importe lequel des cas suivants :

(•) Manuscrit reçu en octobre 1959.
1. Ecoulement non uniforme, et variations de la pente de la ligne d'eau entre de larges limites, même pour une profondeur d'eau constante.

2. Débit présentant des variations et des fluctuations continues; étant donné les variations subites qui se produisent, on ne peut accepter les résultats obtenus au moyen d'appareils de mesures isolés comme valables, mème pour la période de mesures.

3. Rugosité et végétation du fond variables, ou bien végétation excessive.

4. Profondeur et profil du lit variables.

5. Mesure par des appareils isolés (par exemple au moyen d'un moulinet à fonctionnement direci) difficile ou impossible dans le lit originel. 
6. Nécessité de mesurer égalenıent des valeurs extrêmes se produisant rarement.

7. Nécessité d'établir, au moyen de quelques mesures sur place, la relation entre la hauteur (profondeur d'eau, différences de niveau) et le débit.

Un ouvrage de mesure de dimensions convenables peut servir aux fins suivantes :

1. Stabilisation de la courbe d'étalonnage;

2. Mise en graphique préliminaire de cette courbe, à titre d'information;

3. Détermination $\mathrm{du}$ débit instantané à tout moment.

Suivant le licu d'implantation, les ouvrages de mesure servent à plusieurs fins :

A. - Sur des petits conrs d'eau naturels, on peut en général obtenir des données utiles sur les ressources en eau, à partir des enregistrements continus de la sonde de mesure d'un appareil enregistreur.

B. - Sur des canaux de drainage, dans lesquels le plan d'eau se trouve exhaussé et la pente de la ligne d'eau est très faible (de 5 à $15 \mathrm{~cm} / \mathrm{km}$ ), c'est-à-dire dans des conditions moins favorables, l'observation continue des données nécessaires à l'établissement d'une courbe de débit fournit encore des résultats acceptables.

C. - Sur les canaux d'irrigation, dans lesquels une distribution équitable des débits est basée sur des données relatives à la consommation réelle, et dans lesquels il est nécessaire non seulement de mesurer les débits aux prises principales et aux points de distribution, mais encore à l'arrivée chez chaque utilisateur, afin d'éviter des calculs laborieux, le débit fourni devrait être mesuré directement en chaque lieu de consommation.

D. - Dans le cas des alimentations indus- trielles, le rôle de l'appareil de comptage est déterminé par les conditions de mise en œuvre.

Compte tenu de la modernisation du ríseau de jaugeage des débits effectuée au cours des dernières années en Hongrie, les principes suivants ont été adoptés pour la mesure des débits $[2,3$, $4,5]$ :

a) Des ouvrages de mesures ont été installés sur de petits cours d'eau naturels en ayant pour objet principal de permettre la détermination des débits fournis par les bassins versants considérés comme représentant un type particulier. Toutes les chutes aménagées à l'avenir $[4,5]$ se. ront étudiées en tenant compte de la nécessité d'effectuer des mesures.

b) Dans les canaux de drainage, on mesurera des débits aux stations de pompage, et dans le cas de grands bassins rersants, aux limites de chaque zone de caractéristiques différentes.

c) L'étalonnage des prises principales a été achevé dans pratiquement tous les réseaux d'irrigation. L'objet de ces étalonnages est de déterminer les courbes d'étalonnage des ouvrages pour les principaux points de distribution. On a I'intention de mesurer les débits fournis aux utilisateurs individuels au moyen d'ouvrages de mesure construits en bloc avec la prise, ou bien spécialement à cette fin. Le problème principal à résoudre est celui de la mesure des débits aux prises principales et à l'arrivée chez l'utilisateur.

Les expériences résumées plus loin dans le présent article ont été faites surtout pendant la mesure de débits d'irrigation tant sur le réseau qu'en laboratoire. Ces expériences peuvent toutefois être utilisées aussi dans d'autres domaines d'exploitation des eaux. En 1958, on a essayé de mettre au point différents types d'ouvrages de mesures pour des petits cours d'eau naturels et pour des canaux de drainage, mais aucunc expérience n'a encore été réalisćc en lıboratoire à ce sujet.

\section{II. - OUVRAGES DE MESURES ÉTUDIES EN LABORATOIRE ET SUR LES LIEUX}

Les caractéristiques individuelles des ouvrages de mesure peuvent se déterminer par les données de base hydrauliques suivantes :

a) Dimensions caractéristiques (largeur au plafond, fruit des berges, profondeur d'eau, pente du radier, diamètre de buse) du canal (ou de la conduite) au lieu de mesure; b) Débits maximaux et minimaux à mesurer et rapport entre ces débits;

c) Perte de charge admissible;

d) Erreur relative moyenne admissible à la limite inférieure des mesures, c'est-à-dire au débit minimal; 
e) Appareil à implanter dans l'ouvrage (limnigraphe, enregistreur de débit, compteur d'eau);

Dans les cas ci-dessus, il a été nécessaire d'étudier les points suivants :

(c) Détermination de la relation entre le débit et la hauteur d'eau pour des ouvrages de mesure non encore étudiés à l'étranger;

b) Perte de charge engendrée;

c) Détermination des phénomènes de remous dans le bief aval;

d) Mise au point d'une méthode simple pour résumer les résultats.

Les résultats définitifs des études correspondantes sont présentés dans la suite du présent mémoire.

\section{a) Tube Venturi constitué de surfaces coni- ques.}

Ce dispositif simple a été mis au point pour la mesure des débits dans des tuyaux normalisés de $\varnothing 250,300,350,500$ et $800 \mathrm{~mm}$ qui sont d'utilisation courante en Hongrie $[3,6,7]$. Un compteur d'eau proportionnel en parallèle a été installé comme appareil d'enregistrement, suivant les suggestions de E.P. Tiszai et L. Kaffka.

La figure 1 a est le plan de construction d'un tube Venturi pour tuyaux de $250 \mathrm{~mm}$ de diamètre, sur lequel ont été effectués des essais.

Les principes du fonctionnement des tubes Venturi sont bien connus. La hauteur différentielle de mestre commande un dispositif de comptage (compteur d'eau classique). Le tube Venturi a été éludié avec des surfaces coniques

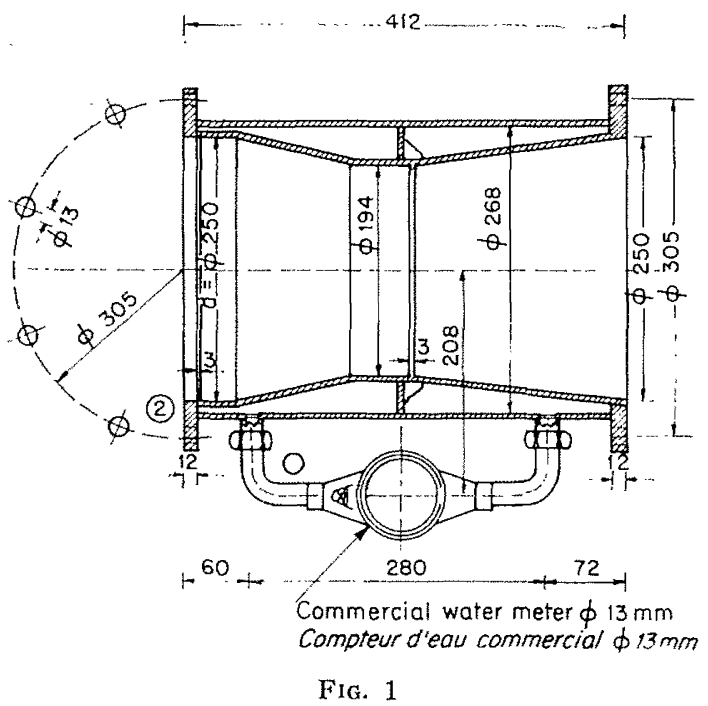
a) Schema; simples et faciles à réaliser dans les fabriques de machines hydrauliques. Les génératrices du convergent forment un angle de $30^{\circ}$ par rapport à l'axe du tuyau, alors que l'angle correspondant pour le divergent est de $16^{\circ}$. Le tube Venturi est disposé à l'intérieur d'un élément de mesure cylindrique (en forme de tuyau) soudé, en tôle épaisse de 3 à $6 \mathrm{~mm}$, et comportant des brides normalisées aux deux extrémités. Les parois coniques du convergent et du divergent sont réalisées en tôle d'acier soudé épaisse de 4 à $6 \mathrm{~mm}$, suivant la taille du Venturi. L'espace vide entre le Venturi et l'élément cylindrique de mesure est divisé en deux chambres annulaires par une tôle de 4 à $6 \mathrm{~mm}$ d'épaisseur. Ces chambres sont en communication avec les zones de haute et de basse pression du tube Venturi, avec la partie parallèle en amont de la contraction et avec la section étranglée, par l'intermédiaire de fentes annulaires.

Le rapport $\left(D_{2} / D_{1}\right)^{2}$ exprimant la relation entre le diamètre $\mathrm{D}_{2}$ de l'étranglement et le diamètre $D_{1}$ du tuyau est de 0,6 dans tous les cas, sauf pour le tube Venturi correspondant au tuyau de diamètre $300 \mathrm{~mm}$; dans ce cas-là, il est de 0,64 .

Les données caractéristiques des tubes Venturi sont résumées dans le tableau ci-dessous :

Le régime d'écoulement à travers un tube Venturi simple pouvant être réalisé avec des surfaces coniques simples a été étudié en utilisant un

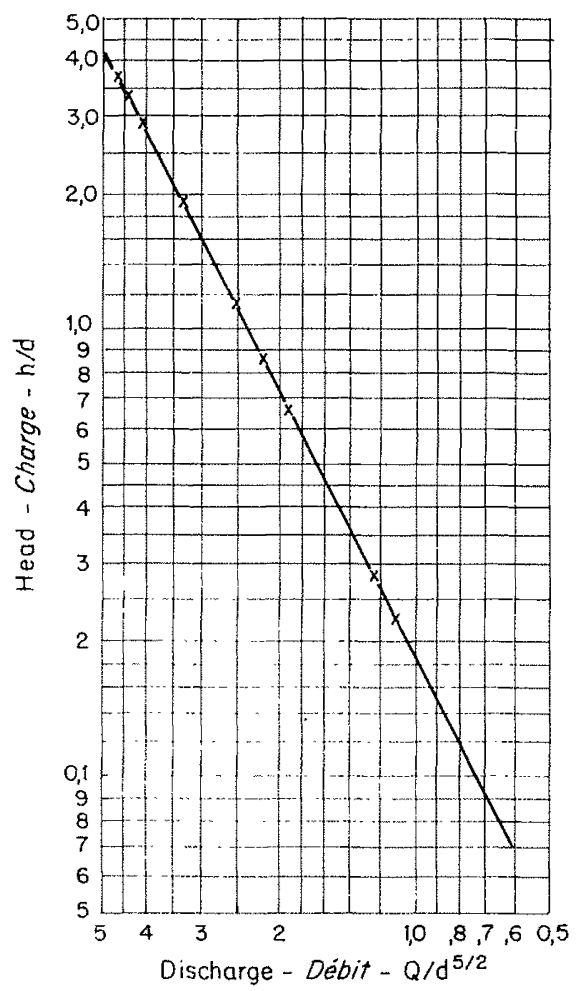

b) Graphique adimensionnel des débits pour les tubes Venturi. 


\begin{tabular}{|c|c|c|c|c|c|c|}
\hline $\mathrm{N}^{\circ}$ & $\begin{array}{c}\text { Diamètre } \mathrm{D}_{2} \\
\text { du tube Venturi } \\
(\mathrm{mm}) \\
(\mathrm{mm})\end{array}$ & $\begin{array}{c}\begin{array}{c}\text { Diamètre } \mathrm{D}_{2} \\
\text { de } \\
\text { l'étranglement } \\
(\mathrm{mm})\end{array} \\
\end{array}$ & $\begin{array}{l}\begin{array}{l}\text { Rapport } \\
\text { de contraction }\end{array} \\
\left(\mathrm{D}_{2} / \mathrm{D}_{1}\right)^{2}\end{array}$ & $\begin{array}{c}\text { Longueur de } \\
\text { l'installation } l \\
(\mathrm{~mm})\end{array}$ & $\begin{array}{c}\text { Distance } \\
\text { du compteur } \\
\text { type MOM } \\
(\mathrm{mm})\end{array}$ & $\begin{array}{c}\text { Coefficient } \\
\text { obtenu } \\
\text { expéri- } \\
\text { mentalement } \\
\mathrm{C}=\mathrm{Q} / q\end{array}$ \\
\hline 1 & 250 & 194 & 0,60 & 412 & 13 & $610-800$ \\
\hline 2 & 300 & 240 & 0,64 & 440 & 13 & $1010-1670$ \\
\hline 3 & 350 & 271 & 0,60 & 522 & 20 & $750-1010$ \\
\hline 4 & 500 & 388 & 0,60 & 800 & 25 & $1010-1500$ \\
\hline 5 & 800 & 620 & 0,60 & 1180 & 13 & $3860-4340$ \\
\hline
\end{tabular}

tuyau d'essai de diamètre $250 \mathrm{~mm}[3,6]$. Les résultats obtenus avec ces essais ont été généraJisés à l'aide du nombre de Froude et en employant des paramètres sans dimensions. Les caractéristiques d'écoulement à travers des tubes de Venturi en similitude géométrique avec celui de diamètre $250 \mathrm{~mm}$ et dont le rapport de contraction est de 0,6; l'angle de convergence de $30^{\prime \prime}$ et l'angle de divergence de $16^{\circ}$, sont montrés dans la figure $1 \mathrm{~b}$ ). Ce schéma peut être utilisé pour la mise au point de tubes Venturi.

Les tubes Venturi montés en parallèle (proportionnels) peuvent être utilisés en combinaison avec des buses ou des conduites de section circulaire, commandées par des vannes. Les règles de base et les conditions pour l'utilisation des tubes Venturi dans les canaux sont les suivantes :

1. Il faut que la différence des hauteurs soit au moins de $5 \mathrm{~cm}$ ou que la vitesse moyenne soit supérieure à $0,7 \mathrm{~m} / \mathrm{s}$ dans le cas d'un tuyau, afin que le débitmètre puisse fonctionner correctement.

2. Il faut prévoir des éléments de tuyau droits sans obstacles, d'une longueur correspondant à au moins quatre fois le diamètre du tuyau, en amont et en aval du tube Venturi.

3. Le compteur doit être installé dans un puits complètement sec et étanche, implanté latéralement et au même niveau que l'axe de la conduite, c'est-à-dire au-dessous du plan d'eau. Afin de protéger le compteur contre une entrée d'air éventuelle, il ne devra pas être implanté au-dessus du niveau des plus basses eaux.

4. Même pour les différences de hauteur maximales, la ligne piézométrique ne doit pas tomber au-dessous du haut du tuyau au droit de l'étranglement, ni au-dessous des orifices prévus pour les prises des tuyaux de mesure situées à la hauteur de l'axe de la conduite.
5. Le niveau minimal aval doit être tel qu'une hauteur d'eau équivalant à un tiers du diamètre de la conduite subsiste au-dessus du bord supérieur de la lumière aval. Pour des vitesses plus faibles (différences de hauteur d'eau de 12 à $15 \mathrm{~cm}$ ), une hauteur d'eau de $5 \mathrm{~cm}$ au-dessus du bord supérieur du tuyau est acceptable.

Il est parfois nécessaire, pour les raisons énumérées ci-dessus, d'installer les tubes Venturi à une plus grande profondeur.

\section{b) Utilisation des coudes pour les mesures.}

Les changements de direction d'un tracé de conduite sont réalisés au moyen de coudes. Il résulte de la répartition inégale des vitesses dans les coudes une différence de pression le long des parois intérieures et exléricures des condes; ces différences peuvent être employées pour faire des mesures sans entrainer de pertes complémentaires. La différence de pression $h$ est proportionnelle à la pression due à la vitesse de l'eau s'écoulant à une vitesse moyenne $v$, c'està-dire :

$$
h=k\left(v^{2} / 2 g\right)
$$

donc, si l'on connaît le coefficient $k$, on peut déterminer le débit d'une conduite en mesurant la différence $h$ des pressions; et cetle différence de pression peut être employée pour faire fonctionner un compteur d'eau (proportionnel) amćnagé en parallèle dans une dérivation.

Il a été établi, sur la base d'expériences réalisées en Hongrie, et dans d'autres pays $[3,7,8]$ que, dans la pratique, le coefficient $k$ est une fonction du rapport $\varepsilon$ du rayon de courbure $R$, mesuré dans l'axe de la conduite, au diametre de la conduite $\mathrm{D}$, 
A. - En admettant que le moment de la quantité de mouvement soit constant, T. Rákóczy a établi pour la valeur de $k$ dans des coudes la relation théorique $[8,25]$ :

$$
k_{\text {théviume }}=\frac{1}{[1-(1 / 2 \varepsilon)]^{2}}-\frac{1}{[1+(1 / 2 \varepsilon)]^{2}}
$$

Les valeurs calculées en utilisant cette expression pour les rapports de courbure $\varepsilon>2$ concordent assez bien avec les résultats expérimentaux; de plus, on a pu calculer des valeurs convenables, en utilisant la formule simplifiée $k=2 \mathrm{D} / \mathrm{R}$, établie par Lansford. Pour des rapports $\varepsilon<2$, les valeurs du coefficient sont obtenues d'après le tableau suivant:

$\begin{array}{cc}\mathrm{R} / \mathrm{D} & k \\ 0,5 & 2,00 \\ 0,8 & 1,97 \\ 1,0 & 1,91 \\ 1,2 & 1,80 \\ 1,4 & 1,64 \\ 1,6 & 1,48 \\ 1,8 & 1,30 \\ 2,0 & 1,14\end{array}$

Lorsqu'on utilise pour des mesures les données expérimentales obtenues avec des coudes lisses, il est important de suivre les règles suivantes :

1. Pour les coudes de $90^{\circ}$, les prises de mesures devraient en principe être situées à la section correspondant à la déviation $\alpha=45^{\circ}$, c'està-dire au centre du coude. Si nécessaire, on peut choisir un angle $\approx$ plus petit $\left(30^{\circ}\right)$. Si l'angle délimité par la section d'entrée et la section dans laquelle sont situées les prises de mesure est inférieur à $30^{\circ}$, le coefficient de hauteur de mesure variera nettement en fonction de la rugosité de la conduite et la distorsion de la répartition des vitesses correspondant à la courbure à travers la section de la conduite se produira à un endroit variant en fonction de la rugosité. Il faut donc éviter l'emploi de coudes dont l'angle central est inférieur a $30^{\circ}$ pour des mesures, sauf, éventuellement, si on les vérifie au préalable par des essais d'ćtalonnage individuel.

2. Les deux prises de mesure doivent être inlplantées aux rayons de courbure minimal et maximal, c'est-à-dire l'une en face de l'autre suivant un diamètre et dans la même section transversale. Les coefficients expérimentaux indiqués ci-dessus conviennent pour ce cas qui, en même temps, correspond à la différence de pression maximale. Cependant, dans le cas où il serait impossible de disposer les prises l'une en face de l'autre, elles peuvent être décalées, mais au prix d'une différence de pression moins élevée. On peut également tenir compte de cette déviation par des calculs théoriques.

3. Le régime d'écoulement dans la conduite doit être turbulent. Les résultats indiqués cidessus correspondent à des nombres de Reynolds $\alpha$ de l'ordre de $5.10^{5}$.

4. Les éléments de conduite en amont et en aval des coudes de mesure ne doivent contenir aucun organe perturbateur, sur certaines longueurs. Des essais effectués pour déterminer la longueur des éléments rectilignes nécessaires pour des coudes lisses et des coudes soudés en éléments séparés n'ont pas fourni des résultats entièrement uniformes. La seule condition qu'il soit donc possible de préconiser à présent est la nécessité d'éviter tout organe perturbateur (tel que d'autres coudes, robinets-vannes partiellement ouverts) sur des longueurs correspondant à 5 diamètres de conduite en amont et 2 diamètres de conduite en aval du coude de mesure. Des coudes au voisinage inmédiat de pompes ne peuvent être utilisés pour des mesures de débit qu'après des essais d'étalonnage individuel, à cause du caractère tourbillonnaire de l'écoulement de l'eau à l'entrée, et ce à condition que le débit ne varie qu'entre des limites relativement étroites. A défaut d'étalonnage individuel, la précision obtenue sera moins bonne. Cette remarque est valable pour tous les cas où il n'est pas possible de prévoir les éléments de conduits rectilignes nécessaires. Dans de tels cas, le coude devra être étalonné dans ses conditions de fonctionnement particulières. De tels étalonnages particuliers sonl également à recommander pour des groupes de pompage de dimensions importantes. Des essais d'étalonnage réalisés au cours de l'étude sur des coudes dont les axes sont situés dans des plans différents ont donné de bons résultats.

5. Des coudes alignés sur le plan vertical entraînent toujours un risque de séparation d'air. Il n'est donc pas recommandé d'installer des compteurs d'eau dans de tels coudes, et tout manomètre utilisé devra être protégé par des réservoir's d'air.

B. - Coudes sondés en éléments séparés. Les stations de pompage en Hongrie utilisent en général des coudes soudés à rayon de courbure irrégulier, et l'on retrouve rarement des coudes lisses dans les installations de pompage provisoire. Aucune mesure du régime des pressions à l'intérieur des coudes soudés n'a encore été effectuce en vue de l'utilisation de tels coudes pour des mesures. Les conclusions présentées ci-dessous ressortent des expériences effectuées par. O. Haszpra au laboratoire de notre Institut avec. des coudes de $250 \mathrm{~mm}$ de diamètre. 
1. La pression différentielle entre les parois intérieures et extérieures des coudes soudés varie de manière continue mais irrégulière. Des maximums localisés ont été constatés aux joints, et des minimums localisés autour des centres des éléments droits. La pression différentielle variait en fonction quadratique du débit.

2. La pression différentielle la plus élevée pour les mesures se produit entre le premier joint le long de la paroi intérieure et le dernier joint le long de la paroi extérieure. Par conséquent, la différence de pression entre ces deux points convient le mieux aux mesures.

3. Comme dans le cas des coudes lisses, la différence de pression entre deux points fixes quelconques du coude peut être ramenée au rayon relatif de courbure, mais au prix de phénomènes localisés plus importants. Dans les coudes constitués de plusieurs éléments droits, le rayon de courbure de l'axe de la conduite est entendu être le rayon du cercle tangent à la ligne brisée.

4. On retrouve toujours des points entre lesquels la différence des pressions est nettement plus importante que dans le cas des coudes lisses du même diamètre. D'autre part, il existe aussi des points où cette différence est plus faible, par exemple au centre des éléments droits.

5 . Les coudes soudés ne peuvent convenir pour des mesures que si les bourrelets de soudure à l'intéricur sont soigneusement lissés au préalable.

6. Les coudes soudés ne conviennent que pour des mesures intéressant des études fournissant des données à titre indicatif seulement. Toutefois, les résultats publiés ci-après peuvent être utilisés pour déterminer à l'avance les différences de pression susceptibles de se produire dans le coude, pour choisir un manomètre approprié, et pour déterminer le débit de la conduite de dérivation. Etant donné que ces coudes sont soudés à la main plutôt que par des méthodes de production en masse, il appartient toujours de les étalonner par des essais spéciaux.

Trois types de coudes ont été étudićs dans le laboratoire (fig. 2). Le coude à $90^{\circ}$ désigné par «A » comprend trois joints à $30^{\circ}$ l'un de l'autre. Le rapport entre le rayon du cercle tangent a l'axe du coude et le diamètre du tuyau est de 2 . O'n a constaté que le coefficient maximal se produisait entre le premier joint intérieur et le dernier joint extérieur, ce coefficient étant de $k=1,875$, alors qu'au centre $d u$ coude, correspondant à un angle de déviation $\alpha=45^{\circ}, k$ était de 1,476 .

l.c coude à $45^{\circ}$ désigné par « $\mathrm{B}$ » comprend deux joints à $22,5^{\circ}$ chacun. Dans ce cas éga- a)
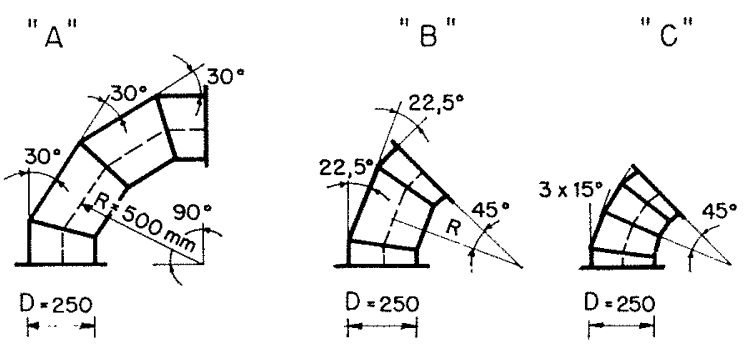

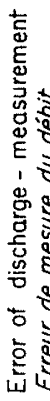

b)

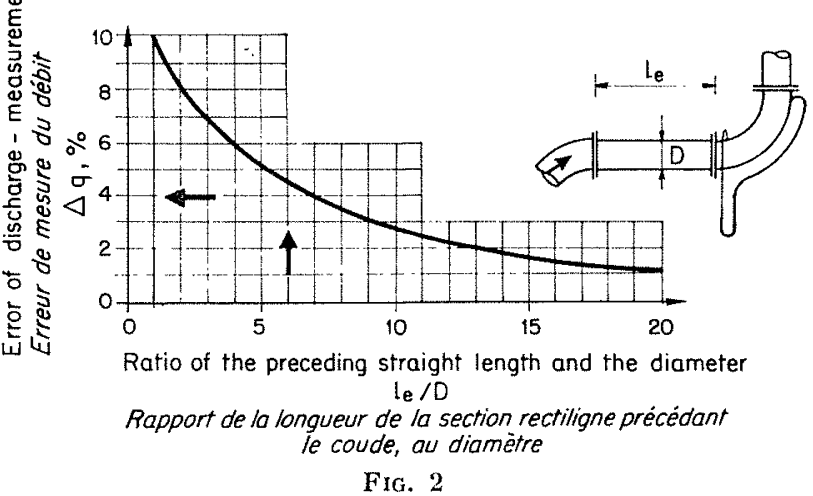

(t) Tuyaux coudés en éléments séparés, qui ont été étudiés; b) Relation entre l'imprécision des mesures dans les coudes étalonnés pour un régime d'écoulement tranquille et la longueur du tronçon de tuyau rectiligne à l'amont du coude.

lemient, le rayon du cercle tangent à l'axe du coude est le double du diamètre du tuyau.

Le coefficient maximal (mesuré entre le premier joint intérieur et le dernier joint extérieur) était de $k=1,647$, alors qu'il était de 0,578 pour les éléments de mesure placés l'un en face de l'autre sur un même diamètre au droit de l'angle central de $22^{\circ} 5$.

Le coude de $45^{\circ}$ désigné par « $C$ » était constitué de trois joints à $15^{\circ}$, représentant approximativement un coude lisse. Le rayon de courbure étail de 1,5. C'est avec des coudes du type «C $\mathrm{C}$ (analogues au coude lisse) que l'on a mesuré la différence de pression maximale au joint central, ce qui donnait un coefficient $k=1,351$.

A la lumière de ce qui précède, l'emploi comme valeur de départ de coefficients de coudes de géométrie analogue à ceux étudiés et illustrés dans la figure 2 , se justifie.

Dans les cas où les angles au joint d'un coude sont identiques à ceux des coudes étudiés, mais où le rapport des courbures est différent, on peut évaluer la hauteur différentielle de mesure à partir de la formule de Lansford. On multipliera 
les valeurs ainsi obtenues par les coefficients suivants :

pour les coudes du type « $A \gg: 1,87$,

pour les coudes du type «B»: 1,65 ,

pour les coudes du type «C»: 1,05 ,

pour obtenir la différence de pression maximale mesurable entre le premier joint intérieur et le dernier joint extérieur.

Les résultats mesurés sont également influencés par la longueur du tronçon de conduite rectiligne précédant le coude composé. On peut constater la manière dont l'erreur de mesure relative diminue en fonction de l'allongement du troncon rectiligne précédant le coude d'après la figure 2, qui est basée sur les résultats expérimentaux. On notera que la présence de phénomènes perturbateurs a généralement donné lieu à une pression différentielle (c'est-à-dire une hauteur: de mesure) plus importante, et, par suite, à une erreur de signe positif.

Dans les cas où la perturbation se trouve au voisinage immédiat du coude de mesure, la relation entre le débit et la hauteur de mesure tend à perdre son équilibre quadratique, et on ne peut s'attendre à des résultats sûrs que si l'on utilise ce coude pour des mesures en cet endroit.

\section{c) Comptage proportionnel par dérivation.}

L'ohtention de données sur les débits de pompage nécessite l'emploi de dispositifs d'enregistrement ou de comptage. On peut remplacer le type d'appareil à dispositif rotatif électrique utilisé présentement ct relativement coûteux, par un compteur d'eau à moulinet, de capacité nominale suffisante, branché en parallèle avec un lube Venturi, ou bien par un diaphragme débitmètre [9]. Les débits passant par le tube Venturi (ou par le débitmètre à diaphragme suivant le cas), installé dans la conduite et le compteur branché en parallèle, sont dans un rapport fixe, de sorte que l'on peut obtenir le débit passant par la conduite principale en multiptiant la quantité enregistrée par le complcur, par un coefficient approprié. Les eaux d'irrigation contiennent souvent en suspension des matériaux solides et des matières fibreuses d'origine régćtale qui ne doivent pas pénétrer dans le compteur. Il appartient donc de prévoir des filtres et des dispositifs de décantation en amont de celui-ci.

L'élément principal d'une telle installation est le bac de décantation [2] à alimentation tangenlielle, dans lequel l'écoulement d'eau ralentit et la plupart des matériaux solides sont décantés. Le rapport de la section de la conduite à celle du bac de décantation est de 1 à 39 . Le reliquat des matériaux solides qui ne se sont pas déposés est arrêté par le filtre à mailles de $1 \mathrm{~mm}$ installè dans le bac. La plupart des éléments de ce dispositif sont nomalisés pour en permettre une fabrication facile, et ils conviennent aussi bien au tube Venturi qu'au diaphragme débitmètre.

La relation entre le débit $Q$ passant par le diaphragme débitmètre (ou tube Venturi, suivant le cas) et la hauteur différentielle $h$ qui en résulte est :

$$
\mathrm{Q}=\sqrt{h / a}
$$

«a» étant une caractéristique constante pour le diapliragme (ou tube Venturi) en question.

Les mesures effectuées sur l'organe expérimen. tal ont indiqué que la relation entre la perte de charge « $h \gg$ à travers le compteur d'eau lorsque celui-ci comportait le filtre illustré dans le schéma, et le débit passant par le compteur d'eau, est :

$$
a=\sqrt{h / 30000000}
$$

Le rapport des débits (c'est-à-dire, en résumć, les volumes d'eau) s'écoulant à travers le compteur d'eau d'une part et la conduite principale d'autre part, est le suivant :

$$
\mathrm{C}=(\mathrm{Q} / q)=\sqrt{30000000 / \alpha}
$$

Dans le cas du tube Venturi et des diaphragmes débitmètres normalisés [2], les valeurs du coefficient $\mathrm{C}$ sont :

Diaphragme de diamètre $80 \mathrm{~mm} \ldots . . \quad 68,3$

$$
\begin{array}{llll}
- & 100 \mathrm{~mm} \ldots \ldots & 107,2 \\
- & - & 150 \mathrm{~mm} \ldots \ldots & 240
\end{array}
$$

Tube Venturi de diamètre $240 \mathrm{~mm}$. . . . $\quad 822$

$$
\begin{array}{rrr}
- & 350 \mathrm{~mm} \ldots \ldots & 1620 \\
- & 500 \mathrm{~mm} \ldots \ldots & 3300
\end{array}
$$

On obtient le débit $Q$ passant par la conduite principale en multipliant le débit indiqué par le compteur d'eau par le coefficient correspondanl (on peut normalement négliger le débit passant par le compteur d'eau).

\section{d) Pertuis de mesure mis au point par Pikalov.}

Le pertuis de mesure mis au point par Pikalov $[10,11]$ consiste essentiellement en un canal de section rectangulaire comportant une vanne du côté aval. Le by-pass alimentant le compteur d'eau situé dans un puits latéral est dérivé du bief amont et rejoint la conduite principale en amont de la vanne. Le débit passant par le by- 


\section{A-A ELEVATION}
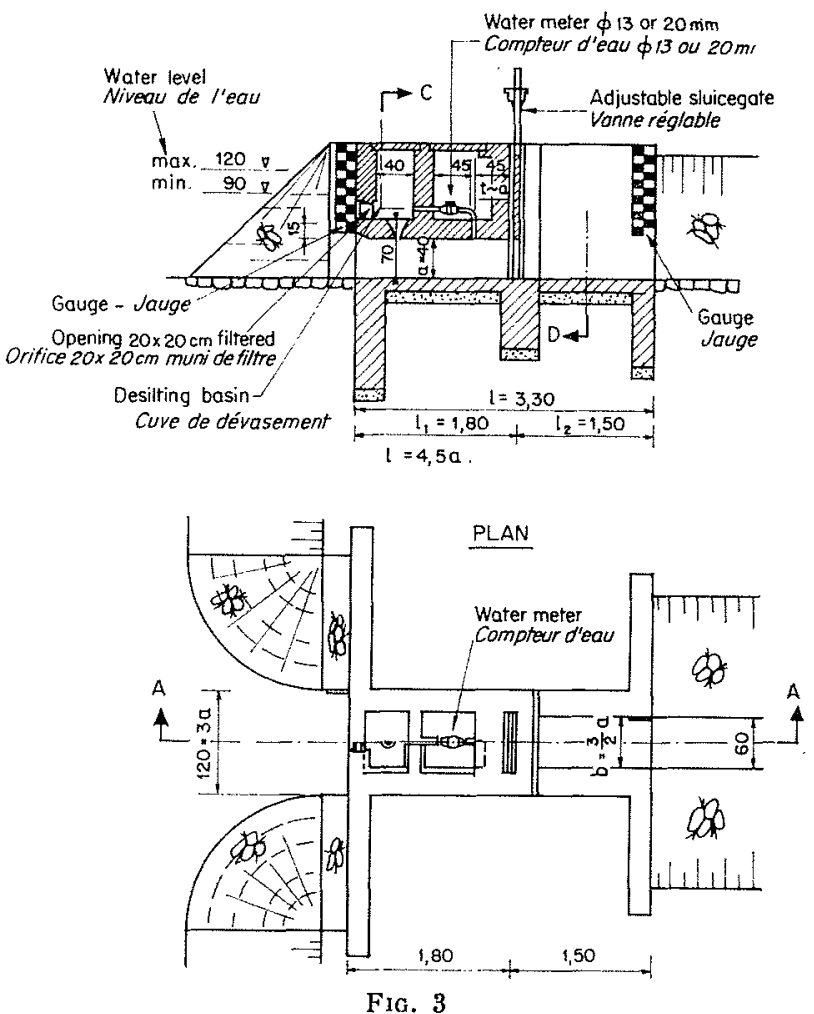

a) Schéma du pertuis Pikalov, modèle hongrois;

pass $q$ est dans un rapport fixe avec celui de la conduite principale $Q$. Dans certains cas, on peut considérer le rapport $q / Q$ comme étant constant et indépendant de la position de la vanne.

La figure 3 montre des schémas d'un pertuis de mesure beaucoup plus court et par conséquent plus économique que l'installation étudiée par Pikalov $[3,6]$. Les résultats obtenus pendant les essais sur modèle dans le laboratoire

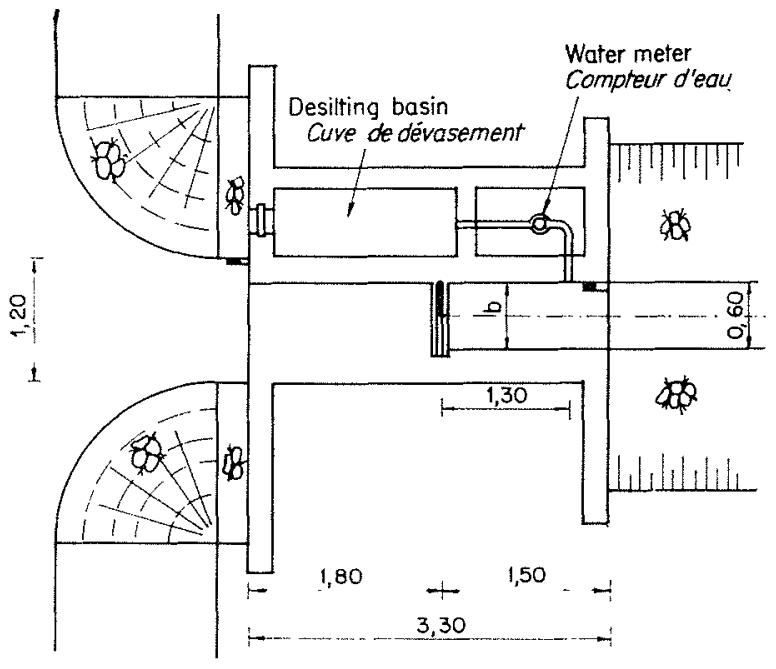

b) Schéma du pertuis de mesures. ont été utilisés pour tracer la figure 4 , dans laquelle les différences entre les plans d'eau aux deux tubes de mesure (hauteur différentielle) sont portés en ordonnée, et le débit en abscisse. Cette figure est établie pour des pertuis de mesure dont le rapport largeur/hauteur est de $2 / 3$. On obtient des résultats légèrement différents si l'on adopte la section transversale $(\mathrm{F}=a b)$ du pertuis de mesure comme troisième paramètre.

Des essais ont montré que le débit est indépendant de l'ouverture de la vanne.

On a employé, pour ces essais, des prises normales à la direction du canal principal; dans une série d'essais, on a fait passer tout le débit par le canal Pikalov, et dans une autre série on a laissé s'écouler une certaine partie du débit en ligne droite, une fraction seulement passant par le pertuis. On n'a pu constater aucune diffé-

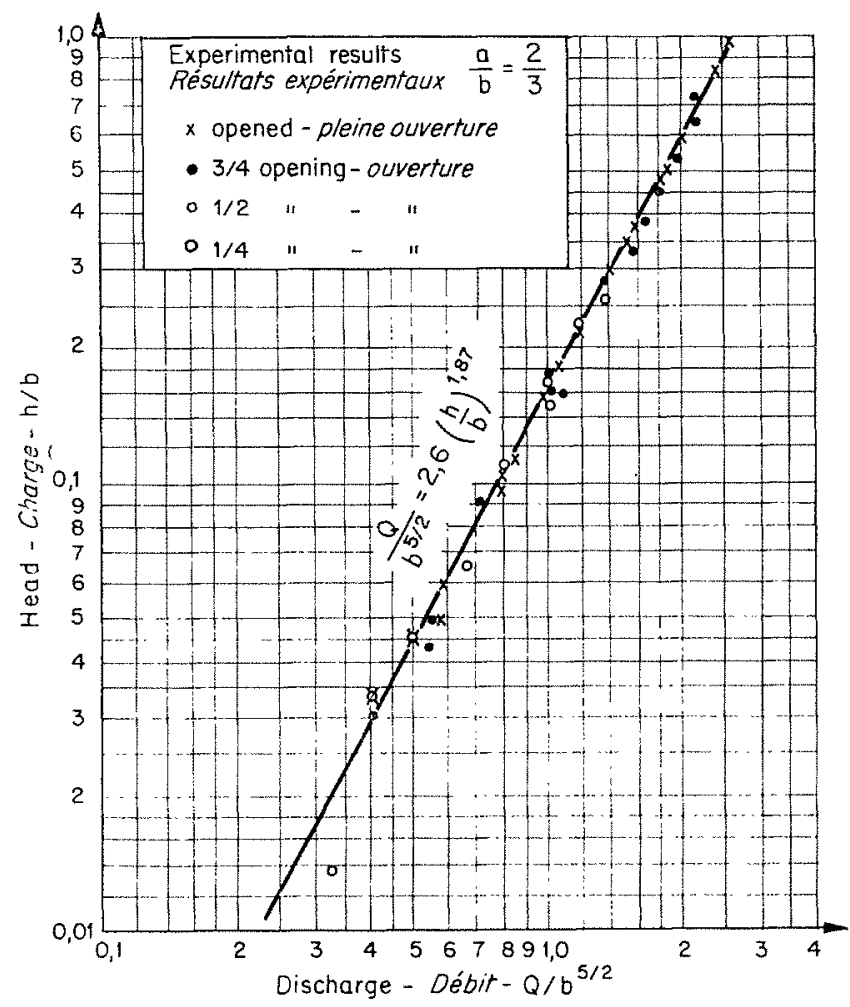

Fig. 4.

Graphique adimensionnel du débit d'un pertuis Pikalov et résultats expérimentaux.

rence importante entre ces deux sortes d'écoulement.

Un inconvénient des pertuis Pikalov est qu'ils ne conviennent que pour la mesure d'une gamme limitée de débits, car pour obtenir une précision normale, les différences de hauteur et de débit ne doivent varier respectivement qu'entre les limites $1: 10$ et $1: 3$.

Lorsque la vanne est partiellement fermée plutôt qu'ouverte en plein, la différence entre les 

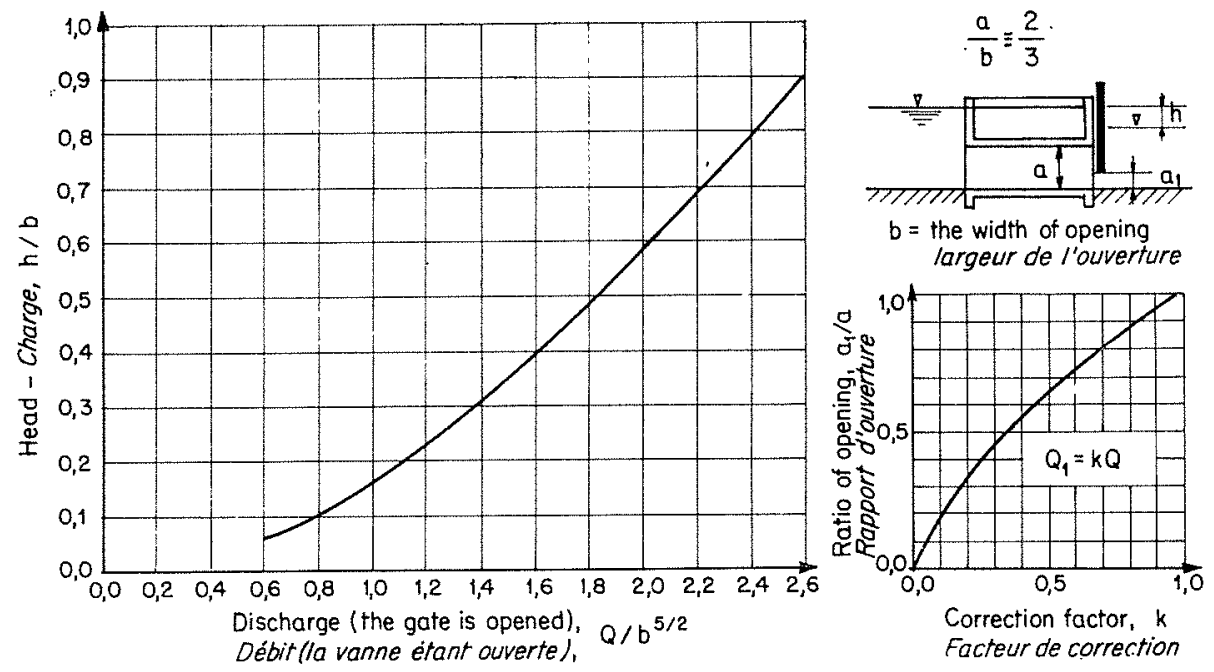

Frg. 5

Graphique adimensionnel du débit des pertuis de mesures.
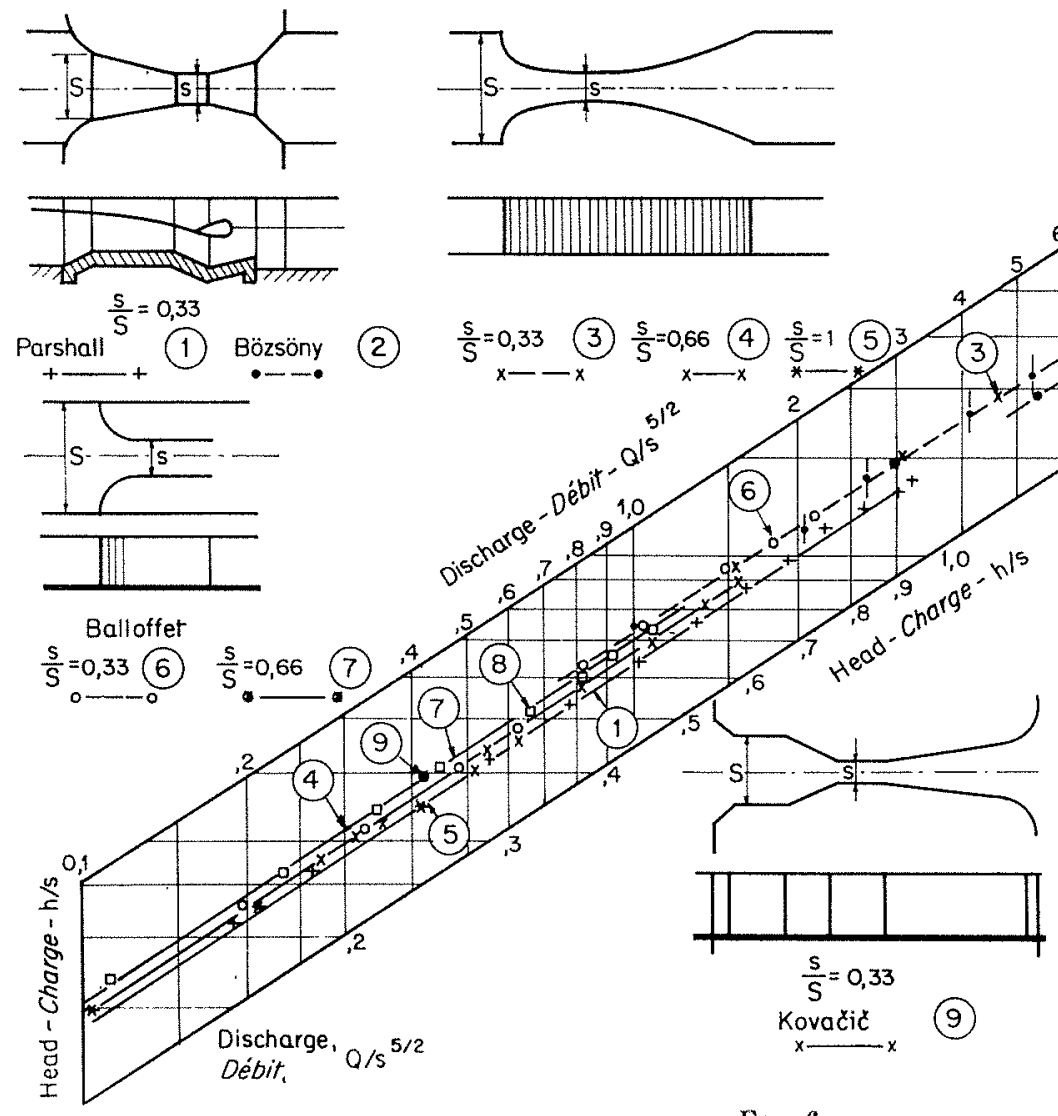

Fig. 6.

Débit des diaphragmes débitmètres. 
plans d'eau amont et aval sera toujours plus grande que la différence des hauteurs aux deux prises de mesure du pertuis Pikalov. Cette plus grande hauteur devrait, en pratique, être utilisée pour les mesures. L'inconvénient principal de l'utilisation de la différence des hauteurs amont et aval pour entraîner un compteur d'eau installé dans un by-pass est que, dans ce cas, le coefficient de multiplication varie en fonction de l'ouverture de la vanne. Cependant, si on limite le nombre des degrés d'ouverture de la vanne à 3 au maximum, et si l'on tient un registre des ouvertures de la vanne, cette solution peut également convenir. Son avantage principal est qu'il est possible d'ajouter à n'importe quel moment un compteur d'eau alimenté par un bypass à n'importe quel ponceau ou ouvrage analogue. La figure $3 b$ montre le schéma d'un pertuis de mesure avec un tuyau by-pass. Les caractéristiques du débit sont données par l'abaque de la figure 5. La valeur obtenue d'après cet abaque doit être corrigée au moyen d'un coefficient (donné par le petit schéma à droite) pour tenir compte de l'ouverture de la vanne. On peut ainsi déterminer les débits correspondant à n'importe quelle ouverture de la vanne. Le coefficient caractéristique du compteur d'eau se détermine par voie expérimentalc en même temps que l'étalonnage du pertuis de mesure.

\section{e) Canaux Parshall.}

Parmi les différents canaux Venturi de section rectangulaire étudiés, la comparaison des résultats d'essai a indiqué la supériorité des canaux Parshall, de plusieurs points de vue. Les canaux Parshall sont des contractions de mesure comportant une section convergente amont, un étranglement incliné vers le bas, et une section aval divergente et inclinée vers le haut. Le bas de l'étranglement se trouve normalement en surélévation par rapport au fond du canal. Les avantages de ces canaux sont les suivants :

1. Capacité de débit augmentée grâce à l'étranglement incliné vers le bas.

Les résultats d'essais, sur modèles, de « contractions de mesure », effectués par Parshall $[12,13]$, de Marchi $[14,15]$, Citrini $[16,17]$, Lamoën [18] et Kovačič [19], ainsi que ceux des essais en laboratoire effectués en Hongrie par Bözsöny [20] sont représentés sous forme adimensionnelle sur l'abaque logarithmique de la figure 6 [21]. (Les dimensions doivent être introduites en mètres.)

La figure montre que, pour des rapports de contraction identiques, les canaux de mesures type Venturi différents possedent approximativement la même capacité de débit, mais celle du

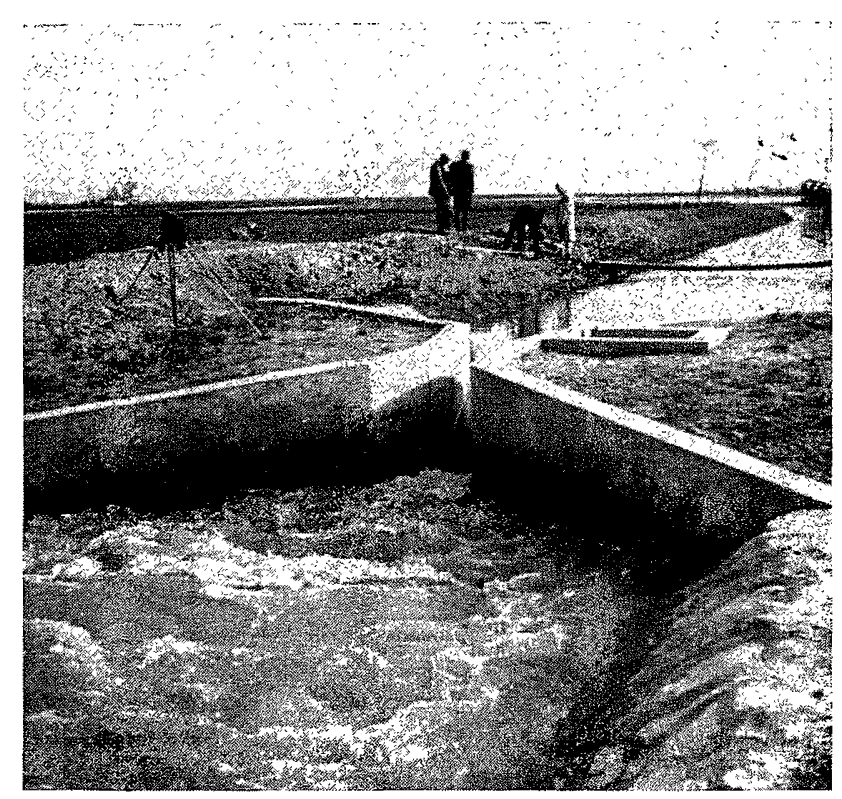

Pното 1

Canal Parshall en fonctionnement.

canal Parshall est la plus élevée si on la ramene au niveau amont (Photo 1). La hauteur différentielle des canaux Parshall doit se mesurer à l'étranglement, plutôt qu'à l'amont, et il a donc été nécessaire, aux fins de comparaison, de corriger les résultats pour les ramener aux niveaux amonts.

2. Pour des débits identiques, les canaux Parshall produisent la plus faible perte de charge. Les expériences de Bözsöny [2, 20], ont montré que la perte de charge des canaux Parshall est inférieure de 10 à $20 \%$ à celle des canaux Venturi à fond lisse.

3. Une grande prudence s'impose en tirant des conclusions générales des résultats d'essais sur modèles réduits à petite échelle, car les erreurs provoquées par des écarts de similitude peuvent atteindre des valeurs excessives, surtoul aux rapports supérieurs à $1: 3$. Toutefois, les données publiées pour les canaux Parshall (13) ont été vérifiées tant par des mesures en laboratoire qu'au cours de campagnes de mesure.

4. Les essais en laboratoire ont, pour la plupart, été effectués dans des canaux d'essai de section rectangulaire, alors que des canaux Parshall ont été vérifiés également dans les canaux trapézoïdaux que l'on rencontre plus couramment dans la pratique.

5. Les coefficients de débit des contractions de mesure sont influencés par le régime d'écoulement immédiatement en amont de l'ouvrage, c'est-à-dire que la perte de charge en amont de l'étranglement est déterminée par les conditions; locales. Par contre, les canaux Parshall ont été 
étalonnés pour une profondeur d'eau correspondant aux deux tiers de l'étranglement et des phénomènes localisés pouvant provoquer un écart de plusieurs points pour cent ont ainsi été éliminés.

$6^{\circ}$ Pour les raisons données dans le paragraphe 5 précédent, de longs biefs amont de section rectangulaire ne sont pas nécessaires et la section trapézoïdale peut s'étendre jusqu'au canal de mesures même.

7. L'ouvrage entier est constitué par des surfaces planes et aucune courbure complexe n'est nécessaire. Le canal peut être réalisé aussi bien en béton qu'en acier ou en bois. L'ouvrage se prête facilement à la normalisation, à un formage normalisé, ou à l'utilisation d'éléments pré-moulés. D'après la littérature publiée à l'étranger, les phénomènes de submersion provenant du côté aval commenceraient à se manifester à partir d'un plan d'eau aval bien défini (par exemple $h_{f} / h_{e}=0,7$ ). Des essais effectués en Hongrie ont indiqué que la limite de submersion serait une fonction du rapport de contraction et de la longueur de l'étranglement $[2,20]$. Des résultats expérimentaux obtenus avec des canaux Parshall sont donnés dans la figure 7 .

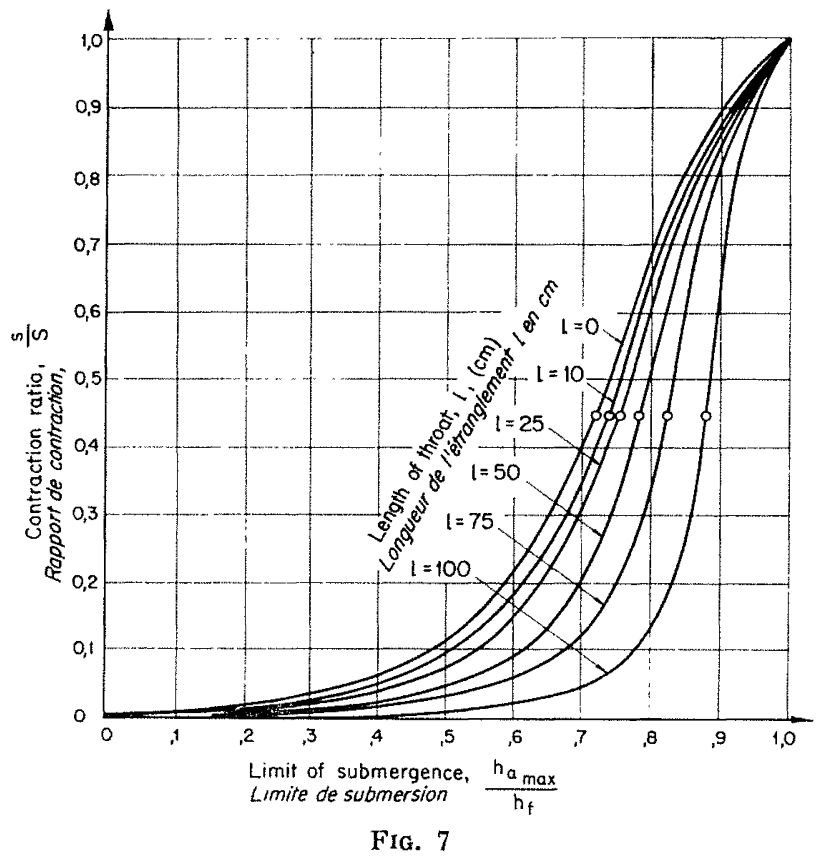

Evolution de la limite de remous.

\section{f) Canaux Venturi trapézoïdaux.}

La gamme des débits mesurables au moyen de canaux du type Venturi de section rectangulaire est nettement plus étendue que celle que l'on peut obtenir avec des dispositifs de mesure à écoulement en charge, mais les canaux

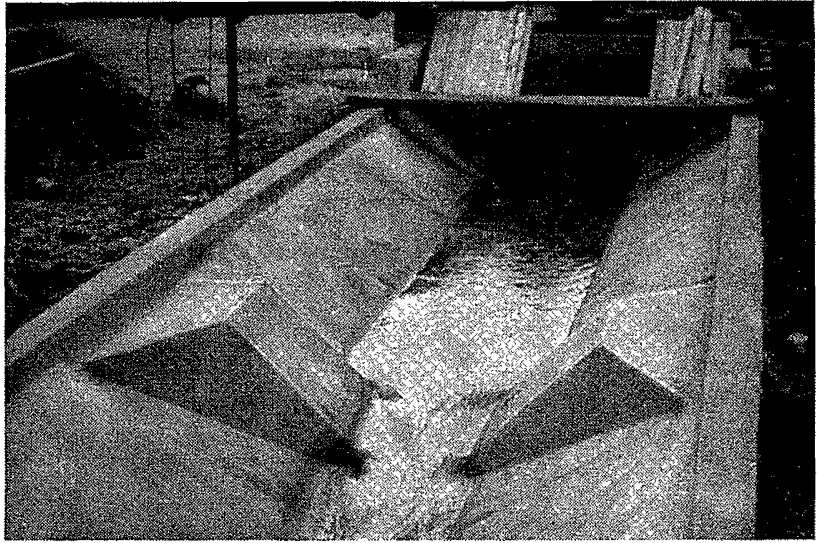

Рното 2

Modèle d'un canal Venturi de section trapézoïdale.

Venturi trapézoïdaux qui permettent une gamme de mesures plus étendue et donnent lieu à une perte de charge augmentant nettement moins rapidement en fonction du débit peuvent également se montrer nécessaires dans certains cas [5]. Plusieurs modèles de canaux Venturi trapézoïdaux ont été étudiés au laboratoire de notre Institut en 1958 (Photo 2). Ces essais en laboratoire ont été réalisés par $O$. Haszpra.

La relation entre le débit et la hauteur différentielle est présentée sous forme adimensionnelle dans la figure 8. Les données caractéristiques des modèles étudiés sont résumées dans le lableau annexé à cette figure.

Il s'est révélé que la limite de submersion n'était pas une constante, mais variait en fonction de la hauteur d'eau.

\section{g) Ouvrages de prise secondaires.}

La méthode la plus économique pour déterminer les débits fournis à ceux qui irriguent est d'utiliser les ouvrages de dérivation ou de prise pour effectuer le jaugeage des débits. Des essais ont été effectués sur les lieux et en laboratoire pour déterminer :

a) les possibilités et conditions dans lesquelles ces ouvrages peuvent être utilisés pour le jaugeage, et

b) Ia capacité de débit en fonction des dimensions du tuyau d'alimentation de l'ouverture de la vanne, et de la hauteur différentielle.

Les expériences en laboratoire effectuées par O. Haszpra ont porté sur des ouvrages de prise de $2 \mathrm{~m}$ et de $4 \mathrm{~m}$ de long et d'un diamètre de $300 \mathrm{~mm}$ comportant des vannes à hausse verti- 


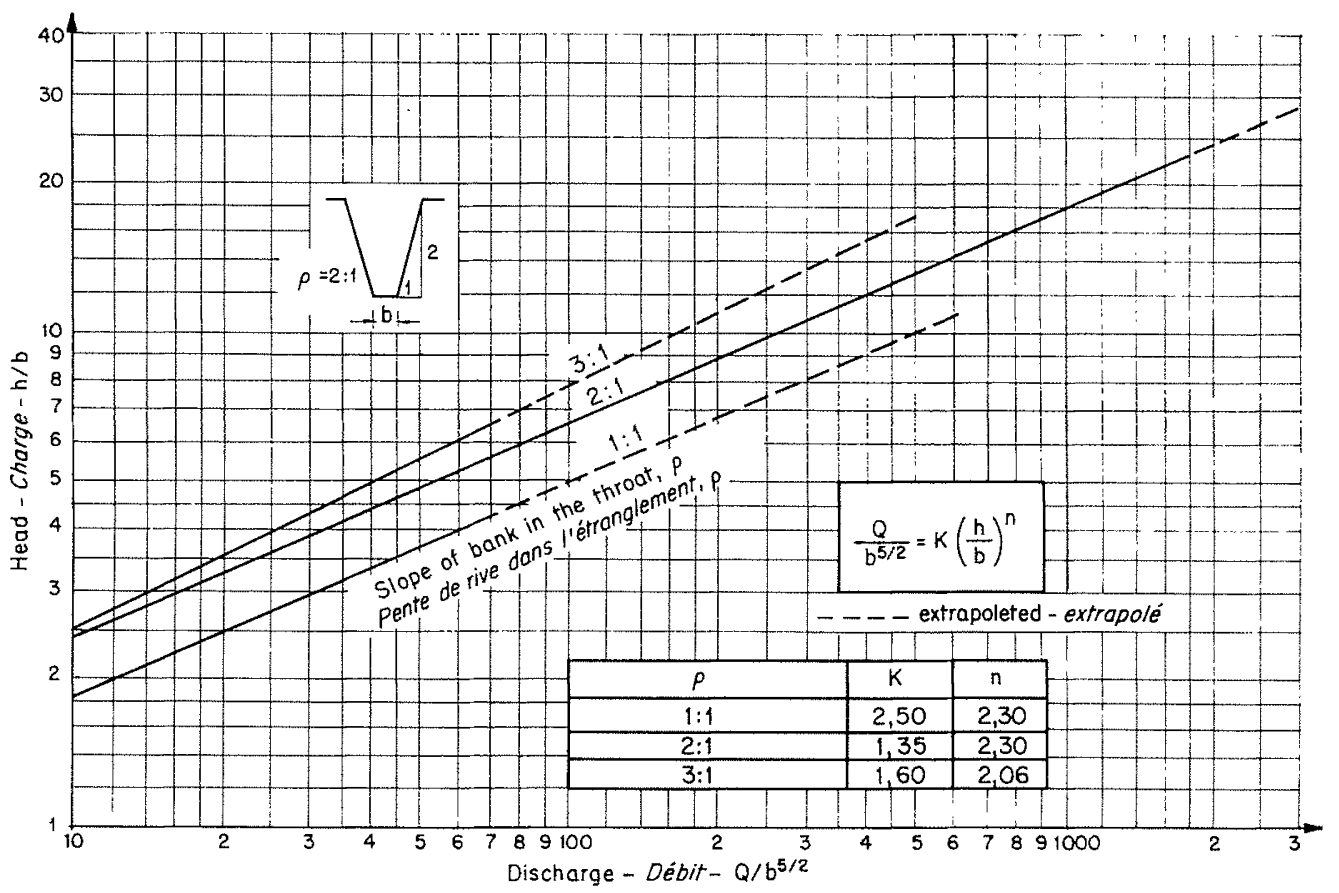

Fig. 8

Débit des canaux Venturi à section trapézoödale.

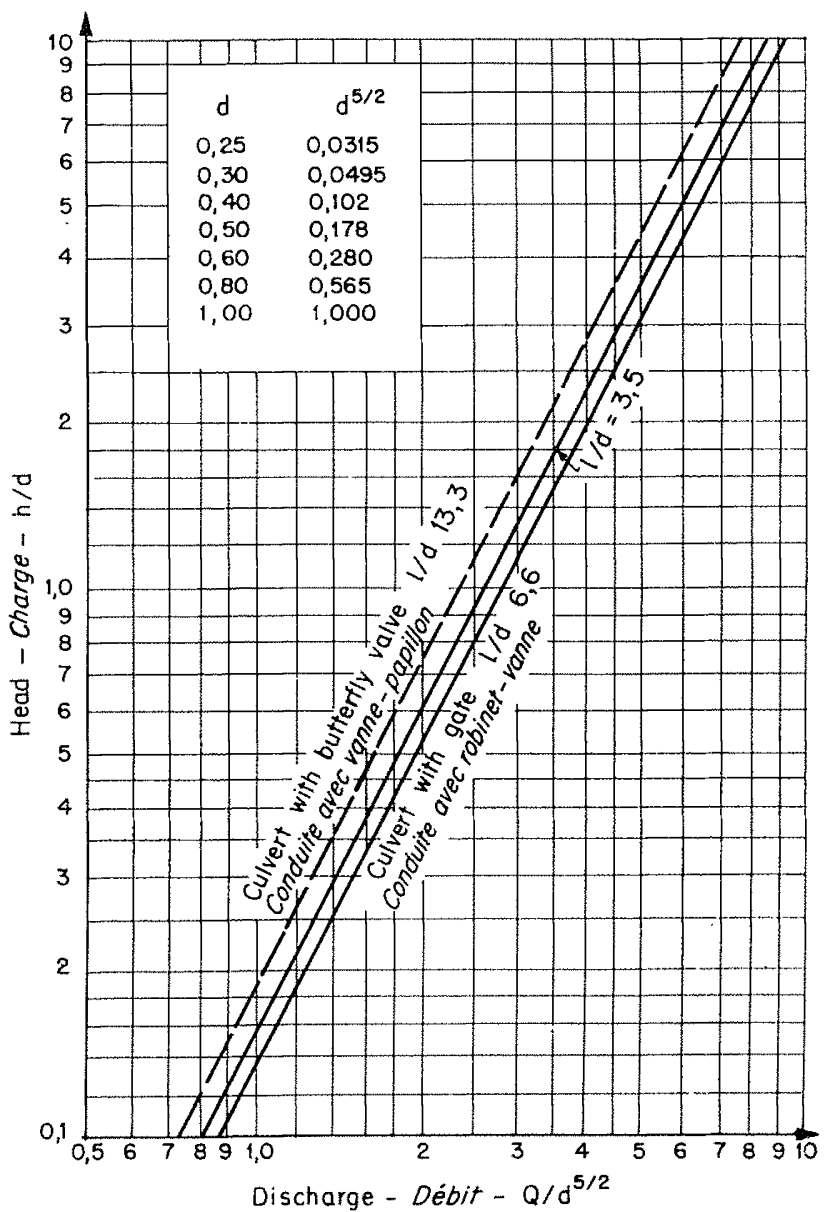

Fig. 9

Débit des pelits ouvrages de dérivation. 
cale, ainsi qu'une prise commandée par une vanne-papillon. On a fait varier la hauteur différentielle de 2 à $50 \mathrm{~cm}$ et le débit de 35 à $150 \mathrm{l} / \mathrm{s}$.

On a pu déterminer au cours de ces essais que seul un débit en charge avec la vanne grande ouverte convenait pour des mesures sûres. Les essais effectués avec la prise à vanne-papillon ont fait apparaître une grande dispersion des résultats quant à la relation entre le débit et la hauteur différentielle à des ouvertures partielles de la vanne.

Les résultats expérimentaux obtenus avec la vanne grande ouverte sont présentés sous forme adimensionnelle dans la figure 9 .

\section{III. - OUVRAGES EXPERIMENTAUX SUR PLACE}

Quelques ouvrages de mesure expérimentaux ont été établis sur le réseau au cours des expériences en laboratoire, tels que :

Un compteur d'eau installé dans une conduite dérivant un pertuis fut essayć en liaison avec l'ouvrage de dérivation des eaux d'irrigation. Un puits, par lequel passait le by-pass sous forme d'un tuyau, était aménagé à côté de la vanne. Pendant l'annce 1957 , le compteur d'eau industriel monté sur le tuyau a fonctionnć avec un filtre, et en 1958, sans filtre. Le coefficient de multiplicalion du compteur d'eau varie en fonction de l'ouverture de la vanne. (Il a heureusement été nécessaire d'utiliser seulement trois ouvertures de la vanne pendant ces opérations.)

Le premier canal Venturi de section trapézoïdale a été construit dans le bassin versant expérimental de notre Institut (Photo 3). Les parois latérales de l'étranglement ont une inclinaison de 3 : 1, la largeur au fond est de $10 \mathrm{~cm}$ et le débit maximal de $2,5 \mathrm{~m}^{3} / \mathrm{s}$. Bien que l'ouvrage ait été étudié pour un régime d'écoulement direct sans

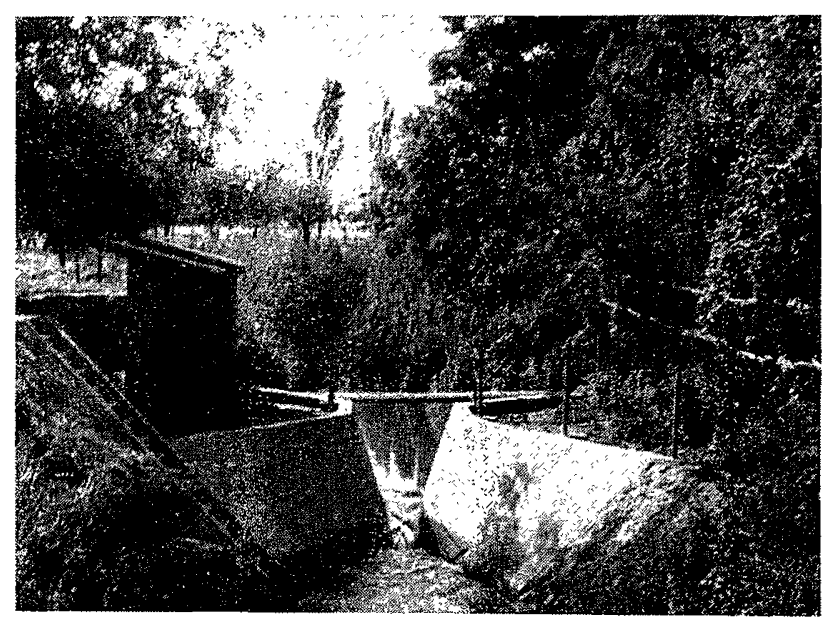

Photo 3

Canaux V'enturi trapézoïdaux. obstruction, on y a adjoint un débitmètre enregistreur à l'aval, étant donné la nature expérimentale de l'installation.

L'étranglement de mesure type Balloffet, figurant dans la photo 4 , et dont le diamètre est de $1,50 \mathrm{~m}$, a été installé dans un petit cours d'eau de montagne en Hongrie [32]. Grâce à sa simplicité exceptionnelle, cet ouvrage convient aux petits cours d'eau à lit profond.

Un des ouvrages de jaugeage les mieux connus, dans les canaux, est le canal à rupture de pente qui, moyennant quelques modifications d'ordre mineur, peut être adapté pour des mesures (fig. 10). Un élément rapporté en béton armé comportant une partie centrale à parois latérales raides est aménagé dans le canal. Au niveau correspondant au débit moyen, la pente de cet élément s'adoucit et devient faible. La partie centrale de l'élément, vers laquelle l'eau est guidée par une surface conique, est normalement de forme trapé. zoïdale, comportant des parois latérales ayant une inclinaison de $1: 1$. Cette section composée fonc-

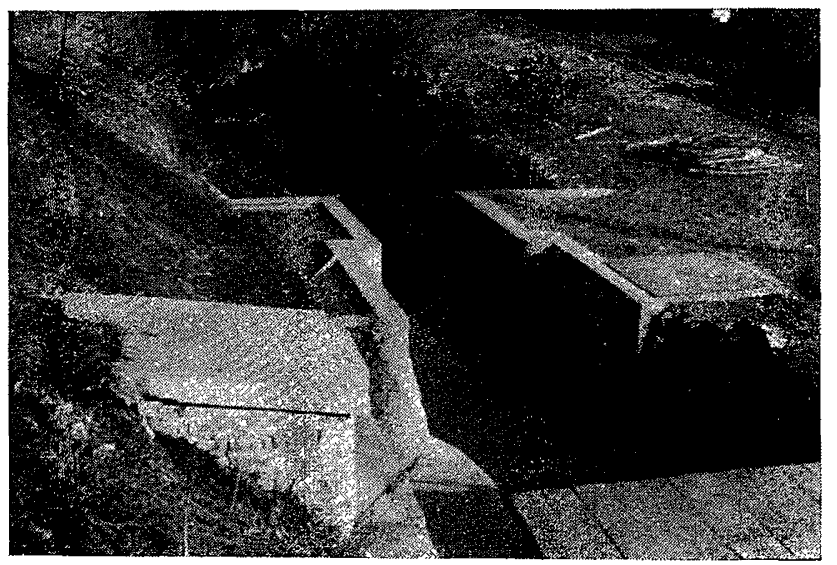

Рното 4

Etranglement de mesure, du type Balloffet. 


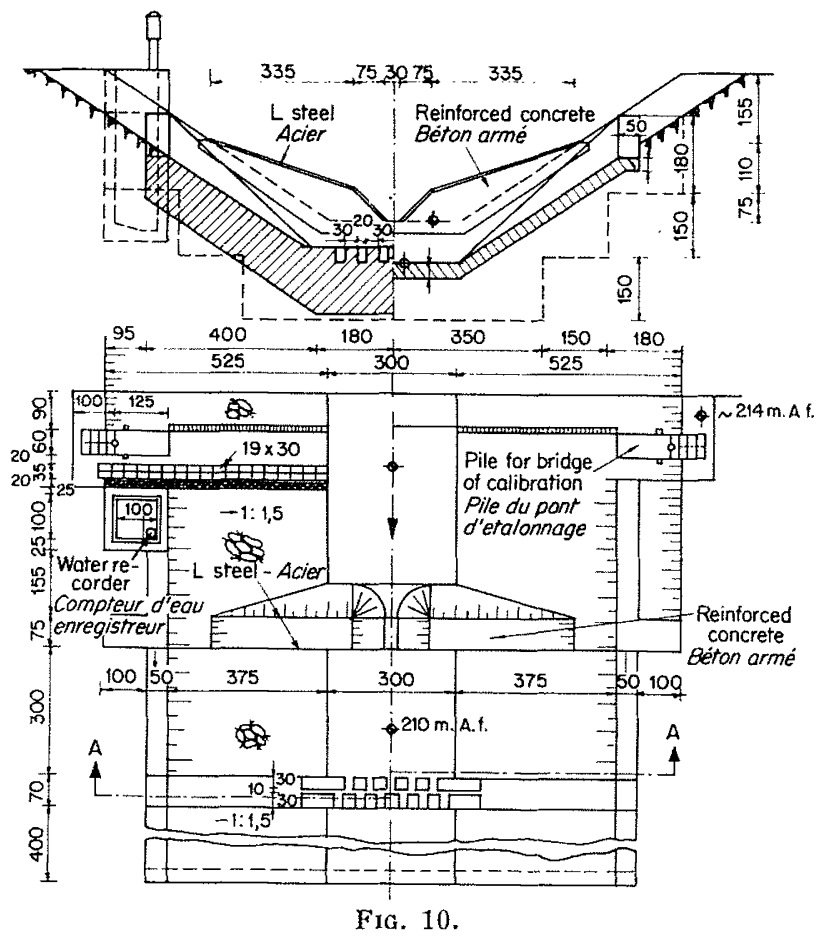

Seuil noyé conçu comme ouvrage de mesure.

tionne comme canal Venturi aux débits d'étiage et comme déversoir à seuil épais aux débits plus importants. Quelques modifications d'ordre mi- neur, nécessaires pour l'étalonnage de l'ouvrage, ont été effectuées, en même temps que son adaptation.

\section{IV. - RÉSUMÉ DES EXPÉRIENCES FAITES EN HONGRIE ET RECHERCHES FUTURES}

Les renseignements tirés des études des méthodes de jaugeage peuvent se résumer de la manière suivante :

1. Les comptages proportionnels avec un simple compteur d'eau peuvent être utilisés sur n'importe quel dispositif ou ouvrage à écoulement en charge (tube Venturi, coudes, pertuis de mesure).

2. Les coudes en éléments soudés peuvent également être utilisés pour les mesures, à condition qu'ils soient installés en position horizontale et qu'ils fonctionnent sous pression.

3. Parmi les divers canaux de section rectangulaire du type Venturi, les canaux Parshall sont les plus favorables du point de vue hydraulique.

4. Il est recommandé d'utiliser des canaux Venturi de section trapézoïdale pour la mesure des débits variant entre des limites étendues.

5. Les ponceaux pour conduites des ouvrages de prise secondaires ne conviennent pas au jaugeage, à moins qu'ils ne débitent en charge et avec leurs vannes grandes ouvertes. Mème dans ces conditions, toutefois, leur précision reste limitée.

6. Les seuils submergés et les chutes sur les petits cours d'eau naturels conviennent bien pour le jaugeage, à condition d'y apporter quelques petites modifications.

Malgré le caractère assez étendu de ces études, il reste encore beaucoup de problèmes qui devront être résolus par des études complémentaires. Ces problèmes sont les suivants :

1. Le comportement des dispositil's de mesure décrits ci-dessus lors de l'utilisation sur le terrain.

2. La possibilité d'utiliser tous les ouvrages normalisés pour le jaugeage.

3. Les comptages proportionnels avec des dispositifs autres que le compteur d'eau industricl (par exemple compteur d'eau du modèle Nikitina).

4. Les intégrateurs automatiques (par exemple, compteur du modèle Gloubshtshev').

5. Les modules et les appareils automatiques de commande du plan d'eau qui leur correspondent. 
Les résultats obtenus jusqu'à présent par l'Institut de Recherches pour les Ressources en Eau ont été publiés $[2,3]$ pour assister les ingénieurs et les utilisateurs en Hongrie.
Il sera possible, sur la base de ces résultats expèrimentaux, de résoudre les problèmes de jaugeage de débits dans tous les domaines de gestion des eaux.

\title{
BIBLIOGRAPHIE
}

\author{
Abréviations : Vituki : Institut de Recherches de Ressources Hydrauliques, Budapest; \\ V.K. : Revue d'Hydraulique, Budapest; Hidrológiai Közlöny : Bulletin de la Société \\ Hydrologique de Hongrie.
}

[1] Muszkalay L. - Measurements and structures rated in 1956 in the irrigation system Tiszalök (1956-ban a tiszalöki öntözôrendšerben végzett mérések és mütárgymtelesitések). Vituki, 1956. Annual Report (Beszámoló).

[2] Karolyi $Z$. and Starosolszky ö. - Measurement of irrigation water (Az öntözõviz mérése). Vituki, 1957 Papers and Report on Research Activities, No. 1, Budapest.

[3] Karolyi $Z$. and Stakosonszky $\ddot{O}$. - Measurement of irrigation water (Az öntözōviz mérése). Vituki, Budapest, 1959. Papers and Report on Research Activities No. 5.

[4] Puskas T. and Stanosolszix $\ddot{0}$. - Modern flow recording stations (Korszerü vizhozam-nyilvántartó szelvények). Vituki, 1957. Annual Report.

[5] Muszkalay L. and Starosolszky Ö. - Flow measurement on small watercourse (Vizhozammérés kisvizfolyáson). Vituki, Publication, 1959.

[6] Fazekas k., Györke A. and Haszpha O. - Laboratory investigations into the measurement of irrigation water ( $\mathrm{Az}$ öntözöviz méréssel kapcso!atos laboratóriumi vizsgálatok). Vituki. 1956. Annual Report (Beszámoló).

[7] Haszpha O. - Results of laboratory experiments into the measurement of irrigation water ( $A$ \% öntözöviz méréssel kapcsolatos laboratóriumi vizsgálatok. Vituki, 1957. Annual Report (Beszámoló).

[8] Rakoczi T. - Flow measurement in elbow pipes (Vizmennyiségmérés könyökökben). Gép, 1954/6.

[9] $\mathrm{F}_{\mathrm{AX}} \mathrm{Cs}$. - Operation of water meters (A vizórảk ïzemtana). Hidrológiai Közlöny, 1954/1-2.

[10] Pikalov, Falukovics and Sapovalopa. - Vodormernoregulirujscsic szooruzsenija i armatura vnutrihozjajsztvennoj araszityelnoj szetyi. Szelholgiz, 1951.

[11] Lesvikov. - Meteorologia, gidrologia i gidrometria. Mosikva, 1955

[12] Parshall R.L. and Rowher C. - The Venturi flume. The Agricultural Experiment Station of the Colorado Agricultural College, Bulletin 265, February 1921.

[13] Parshall. - Measuring water in irrigation channels with Parshall flumes and small weirs. Soil Conservation Service, No. 843, U.S. Dep. of Agricultur., May 1950.

[14| DE Marchi G. - La misura della acque di irrigazione. Memoria e Studi dell' Istituto di Idraulica e Costruzioni Idrauliche, Milano, 1940, No. 38.

[15] DE Marchi G. - Dispositivi per la misura della portata desi canali con minime perdite di quota. L'Energia Elettrica, 1936, Gennaio.

[16] Citroni D. - Misuratori a risalto. Rassegna di alcuni tipi finora adattati. Memoria e Studi dell' Istituto di Idraulica e Costruzioni Idrauliche del Politecnico di Milano, No. 35, Milano, 1939

[17] Citnini D. - Modellatori a risalto. Guida al progetto, 1941.
[18] Lamoïn J. - Note sur quelques types de canaux Venturi. Annales des Travaux Publics de Belgique, juin 1947, Bruxelles.

[19] Kovač č K. - Hidravlično dimenzionranje Venturijevega žleba. Vodogradbeni Laboratorij pri Tehniski Faliulteti Univerze v Ljubljani, Publikacija 1, Ljubljana, 1955, December.

[20] BözsöNY D. - Experiments with Venturi-flumes (Kisérletek a Venturi csatornával) Hidrológiai Közlöny, 1957/2, Budapest.

[21」Starosolszkx $\ddot{u}$. - Choix et dimensionnement du dispositif de jaugeage de l'irrigation. Paper presented before the Second Session of the Yougoslat Hydraulic Association, in Bled, 1958.

[22」 Central Board of Irrigation and Power. - Fluming, Publication No. 6, New Delhi, Revised edition 1957.

23」 Thomas Ch. W. - Structures and methods for measuring irrigation water. Third Congress on $1 T$ rigalion and Drainage, San Francisco, 1957. Reports for discussion, Question g, Part I-R, I LR 18, New Delhi, 1957.

24] Bureau of Reclamation. - Irrigation water measurement. Tables and diagrams. Denver, 1953.

[25] Rakóczi T. - Measurement of flow in pipelines by elborv pieces (A csõvezetékeen áramló folyadékmennyiség mérése könyökcsõvel). Building Installations, $1957 / 1$.

[26] Starosolszky $\ddot{u}$. - Selection of the equipment for the measurement of irrigation water (Az öntözóvizmérõ berendezés megválasztása). Vituki, 1957. Annual Reports.

271 LASZLófFX W. - Flow measurement with fixed weir (A bukõgáttal való vizhozammérés). V.K. 1951/2.

[28] NÉMETH E. - Hydrology and Hydrometry (Hidrologia és hidrometria). Tankönyvkiadó, 1954.

[29] Oroszlany I. - Automatic water distribution in irrigation systems (A vizelosztás automatizálása öntözörendszerekben). Hidrológiai Közlöny, 1955/ $7-8$.

[30] Szesztay K. - Flow measuring and control equipment in Soviet irrigation networks (A szovjet öntözöhálózatok vizhozammérö és szabályozó berendezései). Y.K., 1952/1.

[31] J JRCEV V.N. - Ekszploatacionnaja gidrometrija. Szelholgiz, Moscow, 1955.

[32] Balloffer A. - Critical flow meters (Venturi flumes). Proc. A.S.C.E., No. 743, 1955.

[33] Remenieras G. - Quelques exemples de stations de jaugeage à déversoir pour petits cours d'eau. Mémoire présenté aux $I I I^{r s}$ Journées de l'Hydralllique. Extrait de Pluie, Evaporation, Filtration et Ecoulement, Grenoble, 1954.

[34] Rugatero C. and Grudici P. - Sui misuratori di portata a grande campo di variabilita. L'Energi Elettrica, 1952 , No. 4, Milano.

[35] Muszkalay L. and Starosolszky Ö. - Water measurement manual. Mï̈szali Könyvkiadó, Budapest, 1959 . 